\title{
ARQUITECTURA SISMO-RESISTENTE: RESPUESTA MORFOLÓGICA
}

\section{(EARTHQUAKE-RESISTANT ARCHITECTURE: MORPHOLOGICAL RESPONSE)}

\author{
Hugo Giuliani, Ingeniero \\ Profesor e investigador del Instituto de Investigaciones Antisísmicas de la UNSJ \\ Virginia Rodríguez, Arquitecto \\ Profesor e investigador del Planeamiento y Hábitat de la UNSJ \\ Colaboradores: \\ María Inés Yacante y Ana María Cámpora, Arquitectos, y Hugo Leonardo Giuliani \\ REPÚBLICA ARGENTINA
}

Fecha de recepción: 19 - IX - 94

$409-10$

\section{RESUMEN}

En informes anteriores (1) y (2) planteamos la necesidad de desarrollar una Arquitectura Sismo-resistente, que posibilite la solución integral del problema sismico de los edificios. Se establecieron los fundamentos, objetivos, principios básicos y metodología para deducir las pautas de compatibilización del diseño arquitectónico con el diseño estructural sismo-resistente. En el presente informe se propone una respuesta arquitectónica desde la morfología, que permite llegar a una forma final ajustada, en el sentido de eliminar toda incompatibilidad respecto de las exigencias o condicionantes sísmicos. Es decir, un proyecto arquitectónico que por su propia morfología satisfaga dichos condicionantes. Una "Forma Final Ajustada" que evita, por sí misma, las engorrosas pautas de compatibilización del diseño estructural con el diseño arquitectónico, transformándolas en respuestas morfológicas a cada condicionante sísmico.

\section{SUMMARY}

In the previous reports (1) and (2) we raised the question of the necessity to develop an Earthquake-Resistant Architecture which would make the integral solution of the seismic problem in buildings possible. We established the grounds, objetives, basic principles and methodology for defining the guidelines to be followed in making the architectural design and structural earthquake-resistant design compatible. The present report brings the proposal of an architectural response from the morphological point of view, allowing us to reach an adequate final form which consists of eliminating all the incompatibilities produced by the seismic demands and determinings factors. in other words, we search for an architectural design which, ouing to its very morphology, is able to satisfy the said conditions; an "Adequate Final Form" which in itself avoids the bothersome guidelines for making the structural and architectural designs compatible, transforming them into morphological responses to each seismic determing factor.

\section{Introducción}

Hemos denominado Arquitectura Sismo-resistente, a una arquitectura comprometida con la necesidad de optimizar los procesos de diseño y materialización de los asentamientos humanos localizados en zonas de alto riesgo sísmico, basada en la compatibilización de las interrelaciones entre sus componentes o subsistemas interactuantes durante la acción sísmica.
De esta manera, queremos superar la idea, existente en la mayoría de los arquitectos e ingenieros, por la que responsabilizan solamente a la estructura resistente de la seguridad sísmica del edificio.

Buscamos así la solución integral al problema sísmico, convencidos de que el solo análisis estructural no es suficiente para asegurar la estabilidad sismoresistente de los edificios. Esta solución integral 
debe partir de concebir al edificio como una globalidad donde todas sus partes o componentes interactúan durante la acción sísmica y, consecuentemente, pueden contribuir positiva o negativamente en la respuesta sismo-resistente. Es decir, se hace necesario el análisis de dichas interrelaciones y su compatibilización a los efectos de evitar un escalonamiento de la capacidad resistente del edificio, que nos pueda conducir incluso al colapso del mismo.

A los efectos de este informe conviene recordar el principio básico y el objetivo de optimización, sobre los que se suscita dicha arquitectura sismoresistente:

\section{Principio básico}

\section{"Los elementos estructurales sismo-resistentes durante la acción sismica deben poder responder con la rigidez, resistencia, ductilidad y sincroniza- ción previstas en el diseño y análisis estructural." Es decir, deben poder ofrecer toda su capacidad sismo-resistente en forma simultánea. Caso contra- rio se produce el escalonamiento de la misma, pudiendo llegarse por esta causa al colapso total del edificio.}

La causas que pueden provocar el escalonamiento de la capacidad sismo-resistente de los edificios y que son responsabilidad del diseño arquitectónico son las siguientes:

\section{Torsión sísmica.}

2. Columnas cortas.

3. Seudo-resonancia.

4. Golpeteo.

5. Pisos flexibles.

6. Cambios bruscos de rigideces en planta y altura.

\section{Objetivo de optimización}

Este objetivo, si bien no se refiere a las causas que provocan el escalonamiento de la resistencia sísmica, es de la mayor importancia porque pretende la optimización de la capacidad sismo-resistente de los edificios. Se puede lograr de dos maneras: una reduciendo las fuerzas sísmicas y otra, aumentando la eficiencia de la capacidad sismo-resistente de los edificios.

- La reducción de las fuerzas se puede obtener de diversas formas, a saber: a) La reducción de pesos se puede obtener mediante el uso de materiales más livianos o disminuyendo rellenos y otras terminaciones pesadas, que no sean esenciales a la finalidad de la construcción.

b) Reubicando los mayores pesos, por ejemplo archivos, piletas, etc., en los niveles inferiores.

c) Evitando la seudo-resonancia: esto implica evitar que el período fundamental del edificio coincida con el período predominante del terreno de fundación.

- En relación a la optimización de la capacidad sismo-resistente ésta se puede obtener adoptando configuraciones apropiadas, objetivo principal de la arquitectura sismo-resistente.

Por este camino hemos logrado importantes avances. En efecto, se establecieron los fundamentos, objetivos, principios básicos, metodología para deducir las pautas de compatibilización y las mismas pautas de compatibilización. Sin embargo, la gran ausente la constituía la respuesta desde la Arquitectura que signifique su propia síntesis frente a las exigencias sismo-resistentes. Lógicamente era necesario un período de maduración que implicara asimilación, comprensión y reflexión. Esta respuesta significa organizar y optimizar todos sus elementos o subsistemas que integran el edificio, para responder positiva y eficientemente al sismo. Es decir, como un ejército disciplinado dispuesto a actuar, con su mayor potencial, organizada y eficientemente frente a su enemigo.

Una arquitectura, hasta ahora inexistente, que surja majestuosamente, con sorprendente claridad, firmeza y transparencia. Una arquitectura que se exprese por sí sola. Una arquitectura que simplifique drásticamente las engorrosas pautas de compatibilización del diseño arquitectónico con el diseño estructural sismo-resistente. Una arquitectura que salve al arquitecto de la dictadura de la ingeniería sismo-resistente, porque su respuesta simplifica y minimiza los complicados conocimientos de ingeniería sismo-resistente, necesarios en el estado actual de la Arquitectura Sismo-resistente.

Esta respuesta arquitectónica se ha formulado desde la morfología con total naturalidad y constituye un modo totalmente diferente del hasta ahora utilizado. 


\section{ESQUEMA TRADICIONAL}

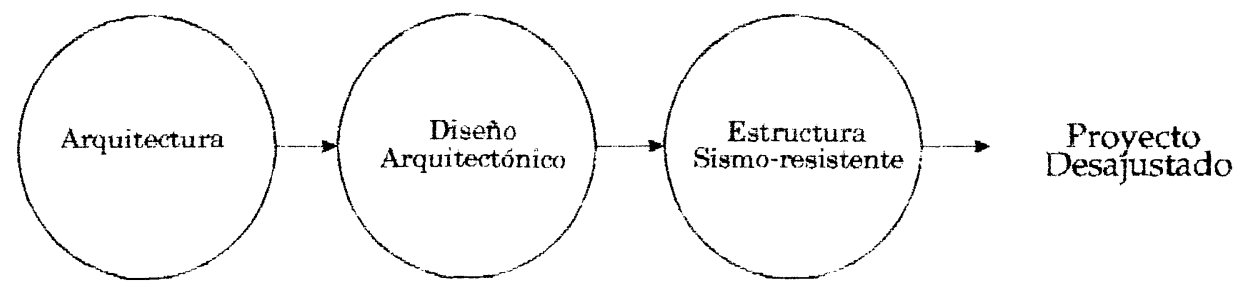

- ESQUEMA DE NUESTRA PROPUESTA INICIAL

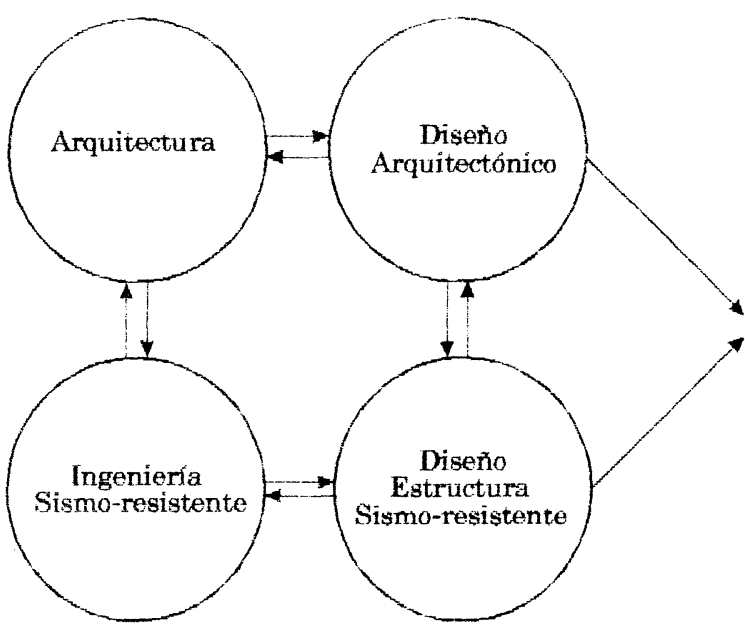

PROYECTO COMPATIBILIZADO

\section{- ESQUEMA DE NUESTRA PROPUESTA MORFOLÓGICA ACTUAL}

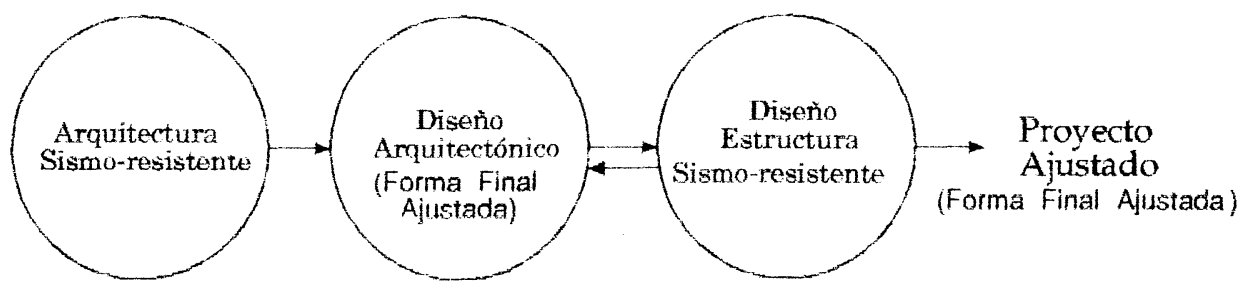

Fig. 1 .

Se trata de establecer pautas o respuestas morfológicas generalizadas a cada condicionante sísmico, tendientes a establecer rápidamente una respuesta morfológica ajustada, que denominamos "Forma Final Ajustada". Esta metodología implica transformar los condicionantes de la ingeniería sismo-resistente en términos de condicionantes morfológicos de fácil interpretación y manejo por los profesionales de la arquitectura.

La Fig. 1 resume esquemáticamente el proceso seguido hasta llegar a la solución morfológica aquí propuesta.
Es de cualquier manera el resultado de una acción interdisciplinaria de Arquitectura e Ingeniería Sismoresistentes.

\section{Forma final ajustada}

La forma final ajustada significa un proyecto arquitectónico del edificio donde todos sus componentes que interactúan durante el sismo lo hacen positiva y eficientemente, optimizando su sismo-resistencia.

La metodología propuesta para el logro de la misma exige transformar todos y cada uno de los condicio- 
nantes sismo-resistentes en condicionantes morfológicos del proyecto arquitectónico en particular.

Este objetivo constituye, sin duda, la base fundacional del método morfológico utilizado. En este sentido comenzamos por establecer las variables referenciales a compatibilizar morfológicamente:

1. Piso flexible.

2. Choque de edificios.

3. Torsión sísmica.

4. Seudo-resonancia.

5. Cambios bruscos de rigideces en planta y elevación.

6. Rigidez-flexibilidad.

7. Pesos concentrados.

8. Columnas cortas.

9. Edificios altos.

10. Edificios con plantas en forma L, U y T.

Corresponde ahora establecer las respuestas morfológicas a cada una de estas variables: en este sentido, los apartados siguientes constituyen un primer intento, que, no obstante, permiten ver la inmediata aplicación de la solución propuesta.

\section{Piso flexible}

Esta situación se da cuando en un determinado piso de un edificio alto, la rigidez del mismo disminuye notablemente respecto a los contiguos.

Esta situación produce una fuerte concentración de fuerzas sísmicas en el mismo, dando lugar a un peligroso mecanismo de escalamiento de la resistencia del edificio.

La respuesta morfológica consiste en evitar esta situación en el diseño arquitectónico. Cuando se requiera un piso de grandes luces, éste deberá ubicarse en el último piso, o ubicarlo fuera de la planta de la torre, y desarrollarlo en un solo nivel preferentemente.

\section{Choque de edificios}

Esto ocurre cuando entre los edificios no existen juntas y el choque se produce cuando sus oscilaciones son desfasadas. Situación completamente normal en estos casos, por lo que se hace necesario evitarla.
La respuesta morfológica consiste en separarlos, tal como prescriben las normas actuales.

En los casos de un mismo edificio, conviene proyectar las distintas funciones en cuerpos separados completamente. Esto evita con seguridad el choque entre los mismos, permite una estructura uniforme y también evita cambios bruscos de rigideces en planta y altura.

\section{Torsión sísmica}

Este efecto ocurre cuando el centro de rigidez (CR) y el centro de torsión (CT) no coinciden. En esta situación aparecen solicitaciones adicionales especialmente en aquéllas más alejadas del CR, pudiendo provocar el escalonamiento de la capacidad sismo-resistente del edificio.

Este problema si bien se tiene en cuenta en el análisis estructural es totalmente indeseable porque genera fuertes fuerzas sísmicas adicionales y en forma desigual entre el conjunto de columnas, dando pie al escalonamiento de la capacidad resistente del edificio.

La respuesta morfológica se logra proyectando edificios con planimetría y altimetría simétricas. Además, es necesaria la simetría estructural, y de los elementos no estructurales interactuantes. Esto exige también la simetría funcional de la planta arquitectónica.

\section{Seudo-resonancia}

Se produce cuando el período propio del edificio coincide con el predominante del terreno de fundación, condición que amplifica notablemente la acción sísmica.

Se compatibiliza haciendo diferentes ambos períodos de vibración, es decir:

$\mathrm{Tt}=\mathrm{Te}=\frac{\mathrm{h}}{100} \sqrt{\frac{30}{\mathrm{Ix}}+2}$ (Fórmula Carmona-Herrera)

donde:

$\mathrm{Tt}=$ período predominante del terreno.

$\mathrm{Te}=$ período propio del edificio .

$\mathrm{h}=$ altura del edificio.

1 = ancho del edificio en la dirección considerada. 
La respuesta morfológica está dada por dicha ecuación ya que Te es función de sus dimensiones espaciales (ancho y altura del edificio). Estas dimensiones habrá que manejarlas para que $\mathrm{T} t \neq \mathrm{Te}$, puesto que $\mathrm{Tt}=$ cte para cada caso particular.

\section{Cambios bruscos de rigideces en planta y elevación}

La eliminación de estas situaciones se logra utilizando, lógicamente, formas espaciales del proyecto arquitectónico compactas, uniformes y homogéneas.

\section{Rigidez-flexibilidad}

Cuando se requiere un edificio rígido o flexible, es decir poco deformable o más deformable se recurre lógicamente a estructuras rígidas, como lo son los tabiques de hormigón armado y o alta densidad de muros de mampostería de alta resistencia y espesor de 0,20 m, o bien todo lo contrario en los edificios flexibles. Sin duda, ambos casos repercuten en la morfología espacial de tales edificios.

\section{Pesos concentrados}

En la mayoría de las actuales normas sismo-resistentes el coeficiente sísmico aumenta casi proporcionalmente a la altura del piso respecto del terreno. En consecuencia, en el Diseño Arquitectónico, es muy importante tener en cuenta este principio, tanto para evitar el uso de materiales pesados, contrapisos, tabiques divisorios, revestimientos, etc., en los niveles superiores, como para ubicar los locales destinados a archivos, piletas de natación o equipos pesados en los niveles inferiores. Se consigue así, no sólo reducir las fuerzas sísmicas por el hecho de que el coeficiente sísmico aumenta en los niveles superiores, sino también reducir, naturalmente, los momentos y cortes sísmicos.

El ejemplo siguiente deja perfectamente aclarada la importancia de este último concepto.

Se trata de una construcción de seis niveles donde se compara el efecto sísmico provocado por un cierto peso $\mathrm{P}$, ubicado primero en el quinto nivel y luego en el primer nivel de la misma construcción (Fig. 2).

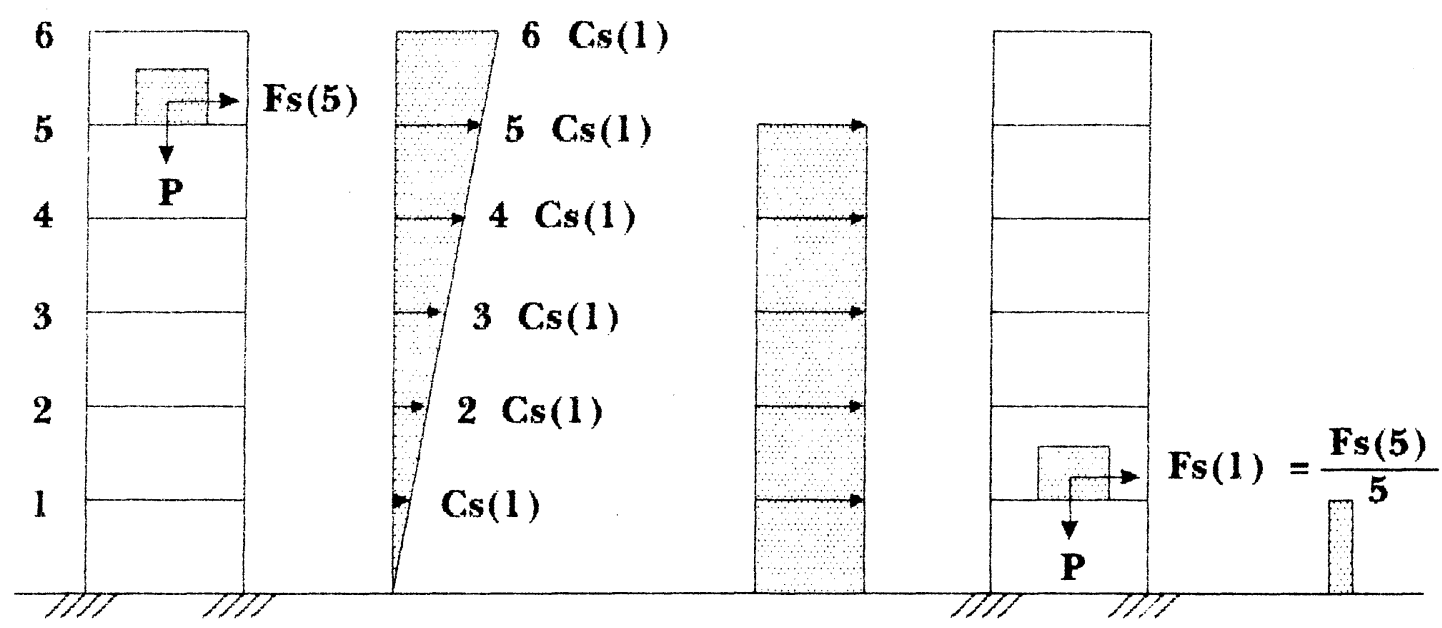

$$
\begin{aligned}
& F s(5)=P \times 5 \operatorname{Cs}(1) \\
& F s(1)=P \times C s(1) \quad \text { luego: } \frac{F s(5)}{F s(1)}=5: \\
& M v(5)=P \times 5 \operatorname{Cs}(1) \times 5 h=25 P \times \operatorname{Cs}(1) \times h \\
& M v(1)=P \times \operatorname{Cs}(1) \times h \quad \text { luego: } \frac{M v(5)}{M v(1)}=25
\end{aligned}
$$




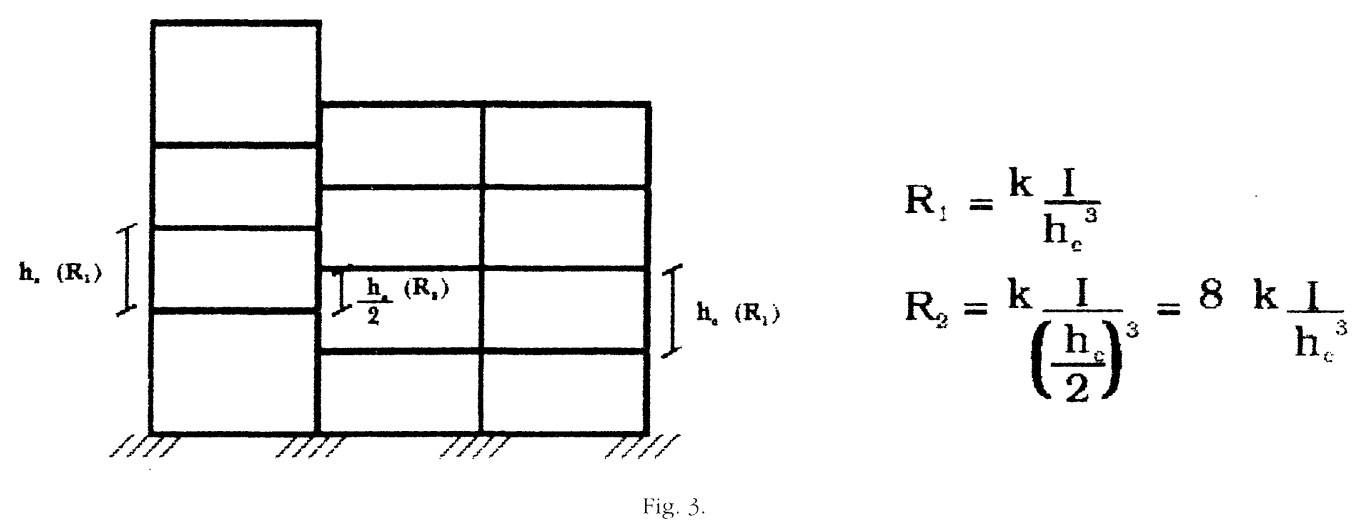

Las conclusiones son terminantes. Para el caso P ubicado en el quinto nivel, el momento al vuelco resulta 25 veces mayor que para el caso de $\mathrm{P}$ ubicado en el primer nivel. Además, el corte sísmico afecta a los niveles del uno al cinco; en cambio, en el segundo caso, sólo al primer nivel y en una relación 5 veces menor.

\section{Columnas cortas}

Otro tema ligado al problema rigidez-resistencia es el denominado "Columna corta". Aquí el corte sísmico aumenta inversamente proporcional al cubo de su altura para columnas de igual área de sección transversal.

Asirivismo, para columnas de muy poca altura esto se arrava por el hecho de que el hormigón no es apic para resistir fuertes esfuerzos tangenciales, disminuyendo notablemente su ductilidad.

Estos casos se producen por una disposición particular de las mamposterías, que reducen la altura de las columnas y consecuentemente su rigidez se incrementa mucho más. Esto hace que se concentre un notable corte sísmico, que lógicamente no puede resistir. La rotura de estos elementos resistentes deja indefenso al resto de los elementos resistentes, pudiendo sobrevenir el colapso total.

Esta situación puede ser fácilmente evitada cuando es consecuencia de la forma y ubicación de las aberturas. En cambio, cuando se debe a desniveles de entrepisos de media altura es prácticamente imposible su eliminación, por lo que estos desniveles deben ser eliminados en el proyecto arquitectónico sismo-resistente (Fig. 3).

\section{Edificios altos}

En los edificios altos (de más de 10 niveles), esta forma ajustada ideal debe exigirse en prácticamente un 100 por ciento.

\section{Edificios con plantas en forma de $\mathrm{L}, \mathrm{U}$ y $\mathrm{T}$}

Las formas L, U y T por la flexibilidad de los entrepisos dan lugar a momentos torsores, aun cuando se haya previsto que $\mathrm{CT} \equiv \mathrm{CM}$. Esto se explica porque la flexibilidad de los entrepisos reduce las rigideces de las columnas, especialmente en los extremos de las correspondientes losas.

\section{Conclusiones}

- La "Forma Final Ajustada" representa, sin duda, el fin último de la Arquitectura Sismo-resistente y también la solución integral del problema sismoresistente de los edificios.

- Su metodología facilita notablemente su aplicación práctica por los profesionales de la Arquitectura y de ninguna manera implica perder originalidad ni mucho menos creatividad. Todo lo contrario, si bien hay que desechar conceptos muy arraigados, permitirá generar otros nuevos y originales que den expresión propia a la Arquitectura Sismo-resistente.

- También es evidente que pretender su aplicación en forma mecánica sin los necesarios conocimientos de diseño estructural sismo-resistente, y en especial de la Arquitectura Sismo-resistente, sí perderíamos originalidad y creatividad y nos conduciría a la situación tradicional.

En los casos de consolidación sismo-resistente de edificios, el primer paso debe dirigirse a una evaluación del diseño arquitectónico a fin de establecer los desajustes de compatibilización de las interrelaciones de las componentes interactuantes durante la acción sísmica. Esta tarea exige profundos conocimientos de diseño estructural sismo-resistente, y en especial de la Arquitectura Sismo-resistente, a fin de 
determinar causas de escalonamiento de la sismoresistencia y sus soluciones modificando, dentro de lo posible, el proyecto arquitectónico y/o estructural.
Es precisamente en estos casos, donde la pretensión de su aplicación en forma mecánico de nada servirá, y caería inevitablemente en defecto.

\section{REFERENCIAS}

(1) Arquitectura Sismo-resistente: "Un Nuevo Enfoque para la Solución Integral de Problemas Sísmicos", Hugo Giuliani y otros. Informes de la Construcción (vol. 38, n. ${ }^{\circ} 387,1987$ ).
(2) "A new approach for the Integral Solution of building design", Hugo Giuliani. Proceeding of the Tenth World Conference on Earthquake Engineering, 1992. Madrid, España.

\section{Publicación del Instituto Eduardo Torroja - CSIC}

\section{Número monográfico de INFORMES}

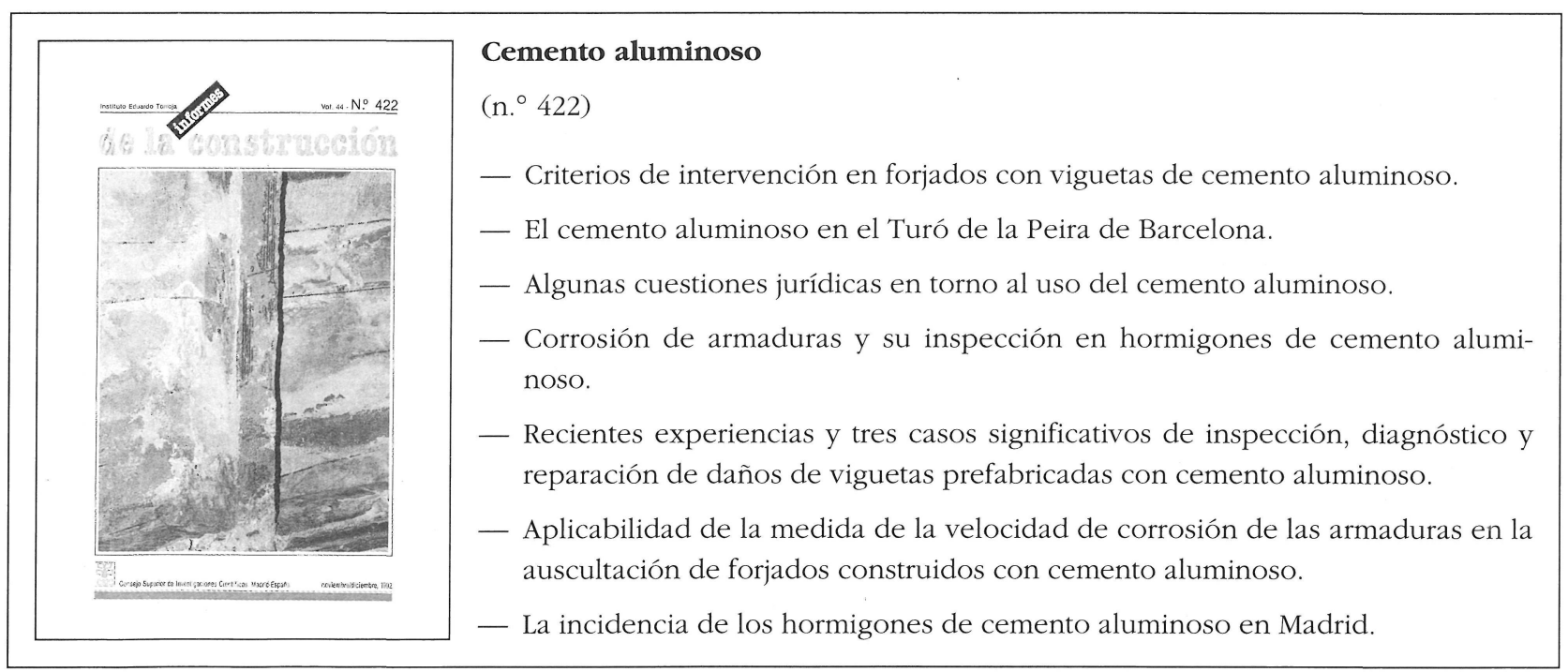

